Mathematical Research Letters 3, 703-721 (1996)

\title{
MULTIPLE GAMMA FUNCTIONS AND $L$-FUNCTIONS
}

\section{ROVINSKY}

Multiple gamma functions were introduced and studied by E. W. Barnes at the end of the last century (cf. [Ba]). It became clear since the time of Barnes, that similar to the case of Dedekind $\zeta$-function, multiple gamma functions are "gamma-factors" of functional equations for Selberg zetafunctions (in the case of double gamma function for an approach based on $[\mathrm{DP}]$ see [Vi], in general [K1]).

On the other hand, multiple gamma functions appear naturally as characteristic "polynomials" of certain differential operators of first order (cf. [Den] for the case of the usual gamma function). In the context of regularized determinants it is possible to define these characteristic "polynomials" correctly and relate them to the Riemann (or Hurwitz) $\zeta$-function.

\section{Notation and conventions}

$B_{n}(x)$ are (resp., $\left.B_{n}=B_{n}(0)\right)$ Bernoulli polynomials (resp., Bernoulli numbers) defined by the power series

$$
\frac{t e^{t x}}{e^{t}-1}=\sum_{n=0}^{\infty} \frac{B_{n}(x)}{n !} t^{n},
$$

(resp.,

$$
\left.\frac{t}{e^{t}-1}=\sum_{n=0}^{\infty} \frac{B_{n}}{n !} t^{n} .\right)
$$

$\zeta(s, u)$ is the Hurwitz zeta function, defined for $\Re s>1$ and $\Re u>0$ by absolutely convergent series $\zeta(s, u)=\sum_{n=0}^{\infty}(n+u)^{-s}$. In fact $\zeta(s, u)$ as a function of $s$ is meromorphic in $\mathbb{C}$, has a simple pole at $s=1$, and $\zeta(1-n, u)=-\frac{B_{n}(u)}{n}$ for all positive integers $n$.

$\zeta^{\prime}(s, u)$ denotes its derivative with respect to the variable $s$.

$\zeta(s)$ is the Riemann zeta function, $\zeta(s)=\zeta(s, 1)=\sum_{n=1}^{\infty} n^{-s}$.

$L(s, \chi)$ is the Dirichlet $L$-function of a (multiplicative) character $\chi$, defined for $\Re s>1$ by absolutely convergent series $L(s, \chi)=\sum_{n=1}^{\infty} \frac{\chi(n)}{n^{s}}$.

$L i_{n}(z)$ is the $n$-logarithm, defined for $|z|<1$ by the power series $\sum_{k=1}^{\infty} \frac{z^{k}}{k^{n}}$.

Received April 10, 1996. 
The Gothic letters are reserved for operators. In particular, $\mathfrak{T}$ will denote a locally unipotent linear operator on the space of polynomials, or on a completion with respect to a topology on it (e.g., the linear operator $f(x) \longmapsto f(x+1))$.

In order to avoid confusion between action of a linear operator $\mathfrak{D}$ on a function $f$ and compositions the operator $\mathfrak{D}$ with the operator of multiplication by the function $f$, let us write them as $\mathfrak{D} f$ and $\mathfrak{D} \cdot f$, respectively.

\section{Definitions}

2.1. Gamma functions. It is well-known that the classical $\Gamma$ function satisfies some very nice properties, such as

- easy behavior under translations by an integer: $\Gamma(u+1)=u \Gamma(u)$;

- Gauß multiplication formula: $\Gamma(u)=(2 \pi)^{-\frac{m-1}{2}} m^{u-1 / 2} \prod_{k=0}^{m-1} \Gamma\left(\frac{u+k}{m}\right) ;$

- $\Gamma(u)$ is a meromorphic function of order 1 of maximal type and all its poles are non-positive integers;

- $\Gamma(u)$ has integer values at positive integer points;

- The complement formula holds:

$$
\Gamma(u) \Gamma(1-u)=\frac{\pi}{\sin \pi u} .
$$

The aim of this section is to define a homomorphism $\Gamma$ from the additive group of the ring of polynomials with integer values at integer points $\mathcal{R}$ to the multiplicative group of meromorphic functions with properties similar to those of $\Gamma(u)$. More precisely, we would like the fraction $\Gamma(P)(u+1) / \Gamma(P)(u)$ to be equal to a product of some powers of $u$ and of $\Gamma(Q)(u)$ for polynomials $Q$ of degree less than the degree of $P$. As a "first approximation" to this homomorphism we can try

$$
\widetilde{\Gamma}: P \longmapsto \widetilde{\Gamma}_{P}(u)=\exp \int_{1}^{u} P(t) d \log \Gamma(t) .
$$

Then

$$
\begin{aligned}
\widetilde{\Gamma}_{P}(u+1) & =\exp \int_{1}^{2} P(t) d \log \Gamma(t) \cdot \exp \int_{1}^{u} P(t+1) d \log \Gamma(t+1) \\
& =\widetilde{\Gamma}_{P}(2) \cdot \widetilde{\Gamma}_{\mathfrak{T} P}(u) \cdot \exp \int_{1}^{u} P(t+1) \frac{d t}{t}
\end{aligned}
$$

where $\mathfrak{T} P(t)=P(t+1)$.

In order to improve this "approximate" definition and make the above formula nice, consider a polynomial correction of the integral in this definition. This appendix is given by a linear operator $\mathfrak{Q}$ on the space of real 
polynomials shifting degrees by one and given by the formula

$$
\mathfrak{Q}=\sum_{n=0}^{\infty} \frac{(-1)^{n+1}}{n !}\left(\zeta^{\prime}(-n)+\zeta(-n, u) \sum_{m=1}^{n} \frac{1}{m}\right) \frac{d^{n}}{d u^{n}} .
$$

Definition 2.1. The homomorphism $\Gamma: P \longmapsto \Gamma_{P}$ assigns to any polynomial $P$ with integer values in integer points, a meromorphic function defined by

$$
\Gamma_{P}(u)=\exp \left[(I d-\mathfrak{V}) \cdot \mathfrak{Q} P(u)+\int_{1}^{u} P(t) d \log \Gamma(t)\right],
$$

where $\mathfrak{V}$ is the operator of value at 1 , and Id is the identity operator.

It will be explained later (see Lemma 3.5 below), why it is a reasonable and in a sense the only reasonable definition of multiple gamma functions. A version of this definition,

$$
\begin{aligned}
& \Gamma_{P}(u)= \\
& \exp \left[\mathfrak{Q} P(u)+\int_{0}^{u} \frac{\widehat{P}(1)-\widehat{P}(t+1)}{t} d t+\int_{u}^{u+1}(\widehat{P}(t)-\widehat{P}(1)) d \log \Gamma(t)\right],
\end{aligned}
$$

where $\widehat{P}$ is a polynomial such that $\widehat{P}(u+1)-\widehat{P}(u)=P(u)$, will be derived later in Corollary 13.

\section{Remarks}

1. This definition coincides with a definition of $\Gamma_{n+1}$ given by Barnes when the polynomial $P(t)=\left(\begin{array}{c}n-t \\ n\end{array}\right)$ is chosen. It will be shown in Corollary 3.3 that

$$
\Gamma_{n+1}(u+1)=\Gamma_{n}(u) \Gamma_{n+1}(u)
$$

for all natural $n$. Clearly, $\Gamma_{1}(u)$ is the usual gamma function.

2. The definition of the multiple gamma function above is given to make it a meromorphic function. However, as it was noticed by Don Zagier, the log multiple gamma functions are more natural. In particular, there is no "global" presentation of the multiple gamma functions as a "period" (like

$$
\Gamma(u)=\int_{0}^{\infty} e^{-t} t^{u} \frac{d t}{t}
$$

and that was the reason, why we did not mention this property among those of the usual gamma function). Another point is that in their connection with various $L$-series, the multiple gamma functions appear only in logarithmized form. Moreover, unlike the (exponential) gamma functions, the $\log$ multiple gamma functions $\log \Gamma_{P}(u)$ can be $p$-adically interpolated to analytic $p$-adic functions (cf. [C-N1]). One more argument is that the 
log multiple gamma functions admit a description as "the finite convolution" of the logarithm and polynomials. This will be explained elsewhere.

2.2. The "sine functions" and the polylogarithms. Once a gamma function is defined, one can symmetrize its definition to get the "multiple sine" function

(2) $\Lambda_{P}(u)=\Gamma_{P}(u) \Gamma_{P^{*}}(1-u)=\exp \left[\mathfrak{Z} P(u)-\pi \int_{1 / 2}^{u} P(t) \cot \pi t d t\right]$,

where $P^{*}(t)=P(1-t)$ and $\mathfrak{Z}$ is a polynomial operator,

$$
d / d u \cdot \mathfrak{Z}=-2 \sum_{n=0}^{\infty} \frac{\zeta^{\prime}(-2 n)}{(2 n) !} \frac{d^{2 n+1}}{d u^{2 n+1}} .
$$

These functions are closely related to polylogarithms.

Classically, the polylogarithm is defined in the unit disc $|z|<1$ by the power series

$$
L i_{n}(z)=\sum_{k=0}^{\infty} \frac{z^{k}}{k^{n}}
$$

Using the obvious identity

$$
L i_{n-1}(z)=z \frac{d}{d z} L i_{n}(z)
$$

one can get an analytic continuation of $L i_{n}(z)$ by means of an iterated integral. For any smooth path $\left.\left.\gamma_{z}:\right] 0,1\right] \longrightarrow \mathbb{C} \backslash\{0,1\}$, joining the origin and the variable point $z$, i.e., $\gamma_{z}(0)=0$ and $\gamma_{z}(1)=z$

$$
\begin{aligned}
\operatorname{Li}_{n}(z)= & \int_{\gamma_{z}} \underbrace{\frac{d z}{z} \circ \cdots \circ \frac{d z}{z}}_{n-1 \text { times }} \circ \frac{d z}{1-z}= \\
& \int_{0 \leq t_{1} \leq \cdots \leq t_{n} \leq 1} \frac{d \gamma_{z}\left(t_{n}\right)}{\gamma_{z}\left(t_{n}\right)} \ldots \frac{d \gamma_{z}\left(t_{2}\right)}{\gamma_{z}\left(t_{2}\right)} \frac{d \gamma_{z}\left(t_{1}\right)}{1-\gamma_{z}\left(t_{1}\right)} .
\end{aligned}
$$

In particular, if the path $\gamma_{z}$ does not cross the ray of reals greater than one, then the corresponding branch of the polylogarithm can be proved to be equal to the following integrals

$$
L i_{n}(z)=\frac{z}{(n-1) !} \int_{0}^{\infty} \frac{t^{n-1} d t}{e^{t}-z}=\frac{z}{(n-1) !} \int_{0}^{1} \frac{(-\log t)^{n-1} d t}{1-t z}
$$

for any $z$ not on the real ray $z>1$. The polylogarithm extends to an analytic function on the whole $\mathbb{P}_{\mathbb{C}}^{1}$ with ramification at $0,1, \infty$. 


\section{Lemma 2.2.}

- Up to a constant multiple, $\Lambda_{P}$ coincides with

$$
\exp \left[-\pi i \int_{0}^{u} P(t) d t+\mathfrak{Z} P(u)+\sum_{k=1}^{\infty} \frac{P^{(k-1)}(u)}{(2 \pi i)^{k-1}} L i_{k}\left(e^{-2 \pi i u}\right)\right],
$$

where $\Im u<0$.

- Up to an exponential multiple any meromorphic function of the type

$$
\exp \left[\sum_{k=1}^{n} f_{k}(u) L i_{k}\left(e^{-2 \pi i u}\right)\right]
$$

for some meromorphic functions $f_{k}$, coincides with $\Lambda_{P}(u)$ for a polynomial $P \in \mathcal{R}$.

Proof.

- For a proof note that logarithmic derivative of $(3)$ is

$$
\begin{aligned}
& \frac{d}{d u} \cdot \mathfrak{Z} P(u)-\pi i P(u)+\sum_{k=1}^{\infty} \frac{P^{(k)}(u)}{(2 \pi i)^{k-1}} L i_{k}\left(e^{-2 \pi i u}\right)- \\
& \sum_{k=0}^{\infty} \frac{P^{(k)}(u)}{(2 \pi i)^{k}} \cdot 2 \pi i \cdot L i_{k}\left(e^{-2 \pi i u}\right) \\
& =\frac{d}{d u} \cdot \mathfrak{Z} P(u)-\pi i P(u)-2 \pi i \cdot P(u) \frac{e^{-2 \pi i u}}{1-e^{-2 \pi i u}} \\
& =\frac{d}{d u} \cdot \mathfrak{Z} P(u)-\pi P(u) \cot \pi u,
\end{aligned}
$$

and coincides with logarithmic derivative of (2) .

- Consider the logarithmic derivative:

$$
\sum_{k=0}^{\infty}\left(f_{k}^{\prime}(u)-2 \pi i f_{k+1}(u)\right) L i_{k}\left(e^{-2 \pi i u}\right) .
$$

It is a meromorphic function. Denote $f_{k}^{\prime}(u)-2 \pi i f_{k+1}(u)$ by $g_{k}(u)$.

Let $g_{N}(u) \neq 0$ and $g_{k}(u)=0$ for all $k>N$. We prove that $g_{k}(u)=0$ for all $k>0$ by induction on $N$, case $N=1$ being trivial. Then

$$
\sum_{k=1}^{N} \frac{g_{k}(u)}{g_{N}(u)} L i_{k}\left(e^{-2 \pi i u}\right)
$$


is a meromorphic function too. Its derivative

$$
-2 \pi i u \frac{g_{1}(u)}{g_{N}(u)} \frac{1}{e^{2 \pi i u}-1}+\sum_{k=1}^{N}\left(\frac{d}{d u} \frac{g_{k}(u)}{g_{N}(u)}-2 \pi i \frac{g_{k+1}(u)}{g_{N}(u)}\right) L i_{k}\left(e^{-2 \pi i u}\right)
$$

should be equal to $-2 \pi i u g_{1}(u) /\left(g_{N}(u)(\exp (2 \pi i u)-1)\right)$ by the induction assumption. Thus

$$
\frac{d}{d u} \frac{g_{k}(u)}{g_{N}(u)}=2 \pi i \frac{g_{k+1}(u)}{g_{N}(u)}
$$

for any $k>0$. Thus for a polynomial $R$ of degree $N-1$ we get

$$
g_{k}(u)=g_{N}(u)(2 \pi i)^{-k} R^{(k-1)}(u) .
$$

Thus

$$
\sum_{k=1}^{N}(2 \pi i)^{-k} R^{(k-1)}(u) L i_{k}\left(e^{-2 \pi i u}\right)
$$

is, again, meromorphic. But the monodromy of the latter about an integer point $m$ subtracts

$$
\sum_{k=1}^{N} \frac{(2 \pi i)^{1-k}}{(k-1) !} R^{(k-1)}(u)(2 \pi i(u-m))^{k-1}=R(m),
$$

and thus, $R(u)=0$, and $f_{k}^{\prime}(u)=2 \pi i f_{k+1}(u)$. Set $P(u)=f_{r}(u)$. Then $P(u)$ is the desired polynomial.

\section{Properties of the gamma functions}

One of the aims of this paper is to show that study of derivatives of Dirichlet $L$-functions is equivalent to study of the values of the multiple gamma functions at rational points. So in all the formulas below the stress will be made on various constants and reducing them to the exponent of the first derivatives of the $\zeta$-function at non-positive integers.

Lemma 3.1. $\Gamma_{P}(u)$ is a meromorphic function of order $\operatorname{deg} P+1$ and infinite type. There is the following decomposition into Hadamard-Weierstra $\beta$ product

$$
\Gamma_{P}(u)=e^{\mathfrak{S} P(u)} u^{-P(0)} \prod_{k=1}^{\infty}\left[\left(1+\frac{u}{k}\right)^{-P(-k)} e^{P_{k}(u)}\right],
$$

where $P_{k}(u)=\int_{0}^{u} \frac{t P(t)+k P(-k)}{t+k} d t$, and $\mathfrak{S}$ is a polynomial operator such that $\mathfrak{S} P(0)=0$ and

$$
\frac{d}{d u} \mathfrak{S} P(u)=\frac{P(0)-P(u)}{u}-\gamma P(u)+\frac{d}{d u} \cdot \mathfrak{Q} P(u) .
$$


Proof. Consider the infinite product presentation of $\Gamma(u)$ :

$$
\frac{1}{\Gamma(u)}=e^{\gamma u} u \prod_{k=1}^{\infty}\left[\left(1+\frac{u}{k}\right) e^{-\frac{u}{k}}\right] .
$$

Take the logarithmic derivative to get

$$
\frac{\Gamma^{\prime}(u)}{\Gamma(u)}=-\gamma-\frac{1}{u}+\sum_{k=1}^{\infty} \frac{u}{(u+k) k},
$$

and multiply by $P(u)$. Then we replace the nominator $u P(u)$ in the $k$ th summand by a constant one, namely by $-k P(-k)$ :

$$
\frac{u}{(u+k) k} \cdot P(u)=\frac{u P(u)+k P(-k)}{(u+k) k}-\frac{k P(-k)}{(u+k) k},
$$

and get the decomposition (4) up to a constant multiple. Due to the formula $(9), \Gamma_{P}(u) u^{P(0)}=\Gamma_{\mathfrak{T}^{-1} P}(u+1)$, so when we multiply both side of (4) by $u^{P(0)}$ and evaluate at $u=0$, we get $1=1$.

The following is the most powerful property of the gamma functions.

Theorem 3.2. Gamma functions are related to the Hurwitz $\zeta$-function by the formula

$$
\Gamma_{P}(u)=\exp \left[\sum_{n=0}^{\infty} \frac{(-1)^{n}}{n !} P^{(n)}(u)\left(\zeta^{\prime}(-n, u)-\zeta^{\prime}(-n)\right)\right] .
$$

Proof. For any $s$ with $\Re s>1$ the series $\sum_{n=0}^{\infty}(n+u)^{-s}$ is absolutely convergent, so one can see the following obvious functional equations:

$$
\begin{aligned}
& \zeta(s, u)=u^{-s}+\zeta(s, u+1) \text { and } \\
& \zeta^{\prime}(s, u)=-\log u \cdot u^{-s}+\zeta^{\prime}(s, u+1) \\
& \zeta^{\prime}(s, u)=-\sum_{n=1}^{\infty} \log (n+u)(n+u)^{-s} \text { and } \\
& \partial / \partial u \zeta^{\prime}(s, u)=s \sum_{n=0}^{\infty} \log (n+u)(n+u)^{-s-1}-\sum_{n=1}^{\infty}(n+u)^{-s-1} .
\end{aligned}
$$

Thus we get an identity

$$
\frac{\partial}{\partial u} \zeta^{\prime}(s, u)=-\zeta(s+1, u)-s \zeta^{\prime}(s+1, u) .
$$


Now take logarithmic derivatives of both sides of (6). Then use the identity (8) and a formula of Lerch

$$
\frac{\partial}{\partial u} \zeta^{\prime}(0, u)=\frac{\Gamma^{\prime}(u)}{\Gamma(u)}
$$

coming from the Bohr-Mollerup theorem (on uniqueness of the function $\Gamma(u)$ such that $\Gamma(u+1)=u \Gamma(u), \Gamma(1)=1$ and $d^{2} / d u^{2} \log \Gamma(u)>0$ for positive real $u$ ).

To see the Lerch formula, specialize $(7)$ to $s=0$. This gives the identities

$$
\zeta^{\prime}(0, u+1)=\log u+\zeta^{\prime}(0, u), \text { and } \exp \zeta^{\prime}(0, u+1)=u \cdot \exp \zeta^{\prime}(0, u) .
$$

The formula (8) above implies

$$
\frac{\partial^{2}}{\partial u^{2}} \zeta^{\prime}(s, u)=s(s+1) \zeta^{\prime}(s+2, u)+(2 s+1) \zeta(s+2, u) .
$$

When $s=0$ this function is reduced to $\zeta(2, u)$, which is real positive for all real positive $u$. Therefore, $\exp \left(\zeta^{\prime}(0, u)-\zeta^{\prime}(0)\right)$ satisfies the assumptions of the Bohr-Mollerup theorem, and coincides with $\Gamma(u)$.

This Theorem has a number of consequences, as can be seen from

\section{Corollary 3.3.}

1. The homomorphism $\Gamma$ commutes with the translation by one in the following way

$$
\Gamma_{P}(u+1)=u^{P(1)} \Gamma_{\mathfrak{T} P}(u),
$$

where $\mathfrak{T} P(t)=P(t+1)$. In particular, for any positive integer $m$

$$
\Gamma_{P}(m+1)=1^{P(m)} \cdot 2^{P(m-1)} \cdot \ldots \cdot(m-1)^{P(2)} \cdot m^{P(1)},
$$

or

$\Gamma_{P}(m+1)=1 !^{P(m)-P(m-1)} \cdot 2 !^{P(m-1)-P(m-2)} \cdot \ldots \cdot(m-1) !^{P(2)-P(1)} \cdot m !^{P(1)}$.

2. "Gauß multiplication formula"

$$
\Gamma_{P}(u)=c_{P}(m, u) m^{\Im P(u)} \prod_{k=0}^{m-1} \Gamma_{\mathfrak{H}_{k, m} P}\left(\frac{u+k}{m}\right),
$$

where $\mathfrak{I} P(u)=\sum_{n=1}^{\infty}(-1)^{n-1} \frac{B_{n}(u)}{n !} P^{(n-1)}(u)^{1}, \mathfrak{H}_{k, m} P(t)=P(m t-k)$ and $c_{P}(m, u)$ is the exponent of a polynomial, the "Gauß exponent". The

\footnotetext{
${ }^{1}$ Notice, that $\frac{d}{d u} \Im P(u)=P(u)$.
} 
latter can be expressed via values of derivatives of the Riemann $\zeta$-function at non-positive integers:

$$
c_{P}(m, u)=\exp \left[\sum_{n=0}^{\infty} \frac{(-1)^{n}}{n !} \zeta^{\prime}(-n)\left(m^{n+1}-1\right) P^{(n)}(u)\right] .
$$

3. Value at $1 / 2$

$$
\begin{aligned}
& \Gamma_{P}(1 / 2)= \\
& 2_{2}^{\sum_{n=1}^{\infty} \frac{B n}{n !} 2^{1-n} P^{(n-1)}(1 / 2)} \exp \left[\sum_{n=0}^{\infty} \frac{(-1)^{n}}{n !} \zeta^{\prime}(-n)\left(2^{-n}-2\right) P^{(n)}(1 / 2)\right] .
\end{aligned}
$$

4. Denote by $\mathbb{Q}\left(\mu_{m}\right)$ the $m$-th cyclotomic extension of the field of rationals. Then the $\mathbb{Q}\left(\mu_{m}\right)$-subspaces of $\mathbb{C}$ generated by

- logarithms of values of gamma functions of order less than $n$ at positive rational points with denominator dividing $m$;

- first derivatives of Dirichlet L-functions with conductor dividing $m$ at integers between $1-n$ and 0

coincide.

Proofs.

1. From an obvious identity

$$
\zeta^{\prime}(s, u+1)-\zeta^{\prime}(s, u)=u^{-s} \log u
$$

valid for $\Re u>0$ and formula (6) we get an identity

$$
\log \Gamma_{P}(u+1)=\sum_{n=0}^{\infty} \frac{(-1)^{n}}{n !} P^{(n)}(u+1)\left(\zeta^{\prime}(-n, u)-\zeta^{\prime}(-n)+u^{n} \log u\right) .
$$

This identity, when combined with the Taylor formula $\sum_{n=0}^{\infty}(-1)^{n} \frac{P^{(n)}(u+1)}{n !} u^{n} \cdot \log u=P(1) \cdot \log u$, gives the identity (9).

2. This follows from an obvious identity

$$
\sum_{k=0}^{m-1} \zeta^{\prime}\left(s, \frac{u+k}{m}\right)=m^{s}\left(\zeta^{\prime}(s, u)+\zeta(s, u) \log m\right) .
$$

3. Plug $u=1$ and $m=2$ into the "Gauß multiplication formula".

4. Apply the formula (6) of the Lemma to $P=(1-2 t)^{n}$ and specialize $u$ to $1 / 2$. This gives a formula for $\zeta^{\prime}(-n)$.

The same formula, applied to $P=(a-m u)^{n}$ describes $\zeta^{\prime}(-n, a / m)$ as a linear combination of logarithms of multiple gamma functions at $1 / 2$ and at $a / m$, and a rational number. The corollary comes as soon as one 
notices that any Dirichlet series with conductor, dividing $m$, is a linear combination of $\zeta(s, a / m)$ for $1 \leq a \leq m$.

\section{Corollary 3.4.}

(13) $\Gamma_{P}(u)=\exp \left[\mathfrak{Q P}(u)-\int_{0}^{u} \widehat{P}(t+1) \frac{d t}{t}+\int_{u}^{u+1} \widehat{P}(t) d \log \Gamma(t)\right]$,

where $\widehat{P}$ is a polynomial such that $\widehat{P}(u+1)-\widehat{P}(u)=P(u)$ and $\widehat{P}(1)=0$.

Proof. Using the formula (9), we get

$$
\begin{aligned}
& \Gamma_{P}(u)=\frac{\Gamma_{\widehat{P}}(u+1)}{\Gamma_{\widehat{P}}(u)}=\exp \left[(\mathfrak{Q} \widehat{P})(u+1)-Q \widehat{P}(u)+\int_{u}^{u+1} \widehat{P}(t) d \log \Gamma(t)\right]= \\
& \exp \left[\mathfrak{Q} \widehat{P}(u)+\sum_{n=0}^{\infty} \frac{(-1)^{n}}{n !} u^{n}\left(\sum_{m=1}^{n} \frac{1}{m}\right) \widehat{P}^{(n)}(u+1)+\int_{u}^{u+1} \widehat{P}(t) d \log \Gamma(t)\right] .
\end{aligned}
$$

From the identity

$$
\begin{aligned}
& \frac{d}{d u} \cdot\left(\sum_{n=0}^{\infty} \frac{(-1)^{n}}{n !} u^{n}\left(\sum_{m=1}^{n} \frac{1}{m}\right) \frac{d^{n}}{d u^{n}}\right)= \\
& \sum_{n=1}^{\infty} \frac{(-1)^{n}}{n !} u^{n-1} \frac{d^{n}}{d u^{n}}: f(u) \longmapsto \frac{f(0)-f(u)}{u} d t
\end{aligned}
$$

we get the identity (13).

\section{Remarks}

1. Strangely enough, differences of the logarithmic derivatives of the gamma function at rationals are algebraic linear combinations of logaritms of algebraic numbers (e.g., [An]). More precisely, for any integers $0<j<$ $N$

$$
\frac{\Gamma^{\prime}(j / N)}{\Gamma(j / N)}-\frac{\Gamma^{\prime}(1)}{\Gamma(1)}=\sum_{1 \neq \zeta \in \mu_{N}}\left(\zeta^{-j}-1\right) \log (1-\zeta),
$$

where $\mu_{N}$ denotes the group of roots of unity of order $N$.

2. A formula of Moreno [Mo] suggests that certain more general multiple gamma functions (also introduced by Barnes) are appropriate to deal with second non-trivial coefficients of Taylor series of $\zeta$-functions of totally real number fields at non-positive integers. However, they can be avoided in the case of totally real abelian extensions of $\mathbb{Q}$. 


\subsection{A universality property of gamma functions.}

Lemma 3.5. For any system of meromorphic functions $\Gamma_{n}$ such that

- $\frac{\Gamma_{n}(u+1)}{\Gamma_{n}(u)}$ is a product of powers of $\Gamma_{k}(u)$ for $1 \leq k<n$ and a power of $u$;

- $\Gamma_{n}(u)$ is a fraction of entire functions of finite order and it has neither zeroes nor poles in a right half-plane;

- $\Gamma_{n}(u)$ is real for positive real $u$ and $\Gamma_{n}(1)=1$,

there exists a unique system of polynomials $P_{n}$ with $\Gamma_{P_{n}}=\Gamma_{n}$.

Proof. By induction on $n$, it is easy to see, that the order of pole (or minus order of zero) of $\Gamma_{n}$ at a non-positive integer $p$ is the value of a polynomial $P_{n}(u)$ of degree $n-1$ at $p$. For $n=1$ this polynomial is a constant, for $n>1$ there is a unique polynomial $P_{n}(u)$ with a fixed value at 0 and such that $P_{n}(m+1)-P_{n}(m)$ coincides with the value at $m$ a fixed linear combination of $P_{k}(u)$ for $1 \leq k<n$ and all negative integer $m$.

Then the fraction

$$
\frac{\Gamma_{n}(u)}{\Gamma_{P_{n}}(u)}
$$

is a periodic meromorphic function of a finite order with no zeroes and poles in a right half-plane, and must be the exponent of a real polynomial of degree $\leq n$ and a rational function of $e^{2 \pi i u}$ simultaneously. So it is the unity.

\subsection{Properties of sine-functions.}

\section{Corollary 3.6.}

1. The homomorphism $\Lambda$, when considered as a function on the product $\mathcal{R} \times \mathbb{C}$, is invariant under the action of the subgroup of even numbers, when $\mathbb{Z}$-action is given by the formula $n:(P(t), u) \longmapsto(P(t-n), u+n)$. More precisely,

$$
\Lambda_{P}(u+1)=(-1)^{P(1)} \Lambda_{\mathfrak{T} P}(u),
$$

where $\mathfrak{T} P(t)=P(t+1)$. In the same sense, it is invariant under the involution $(P(t), u) \longmapsto(P(1-t), 1-u)$ :

$$
\Lambda_{P}(1-u)=\Lambda_{P^{*}}(u)
$$

where $P^{*}(t)=P(1-t)$.

2. "Gauß multiplication formula"

$$
\begin{aligned}
& \Lambda_{P}(u)= \\
& \exp \left[2 \sum_{n=0}^{\infty} \frac{\zeta^{\prime}(-2 n)}{(2 n) !}\left(m^{2 n+1}-1\right) P^{(2 n)}(u)\right] \prod_{k=0}^{m-1} \Lambda_{\mathfrak{H}_{k, m} P}\left(\frac{u+k}{m}\right),
\end{aligned}
$$


where $\mathfrak{H}_{k, m} P(t)=P(m t-k) .{ }^{2}$

3. Value at $1 / 2$

$$
\Lambda_{P}(1 / 2)=2^{-P(1 / 2)} \exp \left[\sum_{n=0}^{\infty} \frac{2\left(2^{-2 n}-2\right)}{(2 n) !} \zeta^{\prime}(-2 n) P^{(2 n)}(1 / 2)\right]
$$

4. Denote by $\mathbb{Q}\left(\mu_{m}\right)$ the $m$-th cyclotomic extension of the field of rationals. Then the $\mathbb{Q}\left(\mu_{m}\right)$-subspaces of $\mathbb{C}$ generated by

- logarithms of values of sine-functions of order less than $n$ at positive rational points with denominator dividing $m$;

- "2 $2 \pi$-normalized" values of Dirichlet L-functions with conductor dividing $m$ at positive integers not exceeding $n$

coincide.

Proof follows immediately from Corollary 3.3.

3.3. The complement formula and fields of sine-functions. Suppose for a moment that we do not know yet what is the function $(\Gamma(u) \Gamma(1-$ $u))^{-1}$. Then we can describe it as the only solution of the differential equation $f^{\prime \prime}=-\pi^{2} f$, with the initial data $f(0)=0$ and $f^{\prime}(0)=1$. There are no that simple differential equation for the functions $\Lambda_{P}$. But the following claim may be considered as a replacement of the complement formula.

\section{Lemma 3.7.}

1. The function $\Lambda_{P}$ is of the same order as $\Gamma_{P}$.

2. Unlike gamma functions, any multiple sine function $\Lambda_{P}$ generates over $\mathbb{C}$ (resp., over $\mathbb{Q})$ a differential field $\Phi_{P}^{\circ}$ of degree of transcendence not more than three (resp., $[(\operatorname{deg} P-1) / 2]+5)$.

If $\operatorname{deg} P>2$, then differential endomorphisms of the field $\Phi_{P}^{\circ}$ over $\mathbb{C}$ form a group isomorphic to one-dimensional algebraic torus $\mathbb{C}^{\times}$, which coincides with the differential Galois group of the field $\Phi_{P}^{\circ}$ over $\Phi_{1}=$ $\mathbb{C}(u, \pi / \sin \pi u)$.

3. Multiple sine functions of order $\leq d$ generate over $\mathbb{C}$ a differential field $\Phi_{d}$ of degree of transcendence $d+1$.

Differential endomorphisms of the field $\Phi_{d}$ form a group isomorphic to a (certainly, non-algebraic) group $\left(\mathbb{C}^{\times}\right)^{d-1} \times \mathbb{Z}$.

Proof.

1. Straightforward.

\footnotetext{
${ }^{2}$ The functional equation for $\zeta(s)$ implies $2 \frac{\zeta^{\prime}(-2 n)}{(2 n) !}=(-1)^{n+1} \frac{\zeta(2 n+1)}{(2 \pi)^{2 n}}$.
} 
2. Immediately from the definition

$$
\frac{d}{d u} \log \Lambda_{P}(u)=-2 \sum_{n=0}^{\infty} \frac{\zeta^{\prime}(-2 n)}{(2 n) !} P^{(2 n+1)}(u)-\pi P(u) \cot \pi u .
$$

Thus a field, generated by $\Lambda_{P}(u), u, \pi \cot \pi u, \pi^{2}$ and $\zeta^{\prime}(-2 m)$ for all integer $m$ between 0 and $(\operatorname{deg} P-1) / 2$, is closed under the differentiation. 3. Since the subfields $\mathbb{C}(u)$ and $\mathbb{C}(\cot \pi u)$ are closed under the differentiation, they should be preserved by any differential endomorphism. Clearly, their endomorphisms form groups isomorphic to $\mathbb{G}_{a}(\mathbb{C})$ and $\mathbb{G}_{m}(\mathbb{C})$, respectively.

The above expansion for the logarithmic derivative of $\Lambda_{P}$ shows that differential endomorphisms should almost preserve the set of poles of $P(u) \cot \pi u$ ('almost' means upto a finite set). Thus they must act as translations by integers on $\mathbb{C}(u)$.

One can visualize the above in the commutative diagram of fields

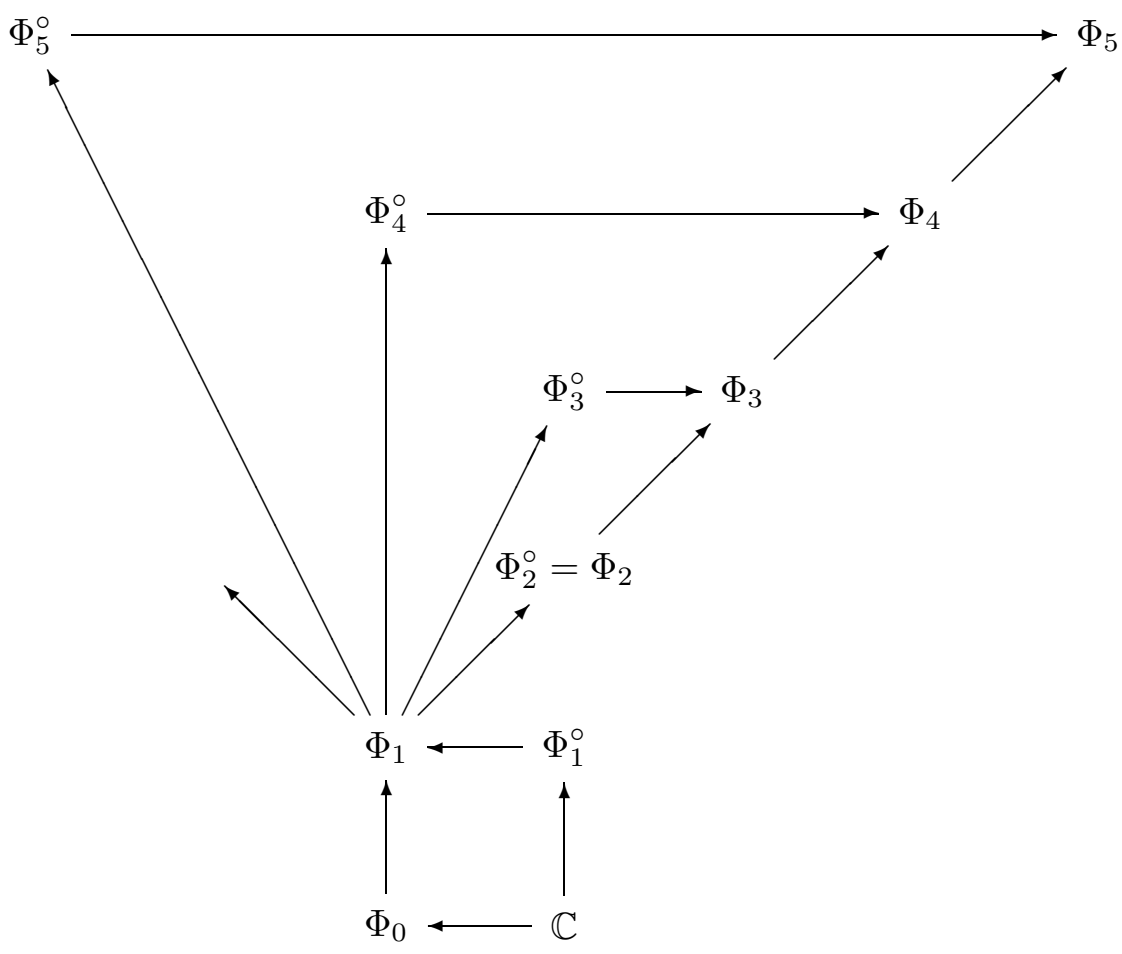


where each non-horizontal arrow is a purely transcendental Picard-Vessiot extension of degree one.

\section{An $L$-function}

Let $Q(t)$ be a monic polynomial of degree $m$ with roots in the left halfplane. Generalizing [Va] (compare, however, with [C-N1], [C-N2],[C-N3]), let us introduce the following $L$-series:

Definition 4.1. For a polynomial $P(t)$

$$
L_{P}(s, Q)=\sum_{n=0}^{\infty} \frac{P(-n)}{Q(n)^{s}} .
$$

The following accounts some basic properties of $L_{P}(s, Q)$.

\section{Lemma 4.2.}

1. $L_{P}(s, Q)$ is $\mathbb{C}$-linear with respect to $P$.

2. For a positive integer $v$

$$
L_{P(t)}(s-v, Q)=L_{P(t) Q(-t)^{v}}(s, Q) .
$$

3. The function $L_{P}(s, Q)$ admits an analytic continuation to the universal cover of the variety $\left\{(s, Q) \in \mathbb{C} \times \mathbb{A}_{\mathbb{C}}^{m} \mid Q(k) \neq 0\right.$ for $\left.k \in\{0,1,2,3, \ldots\}\right\}$ with simple poles along the divisors $s=-k / m$, where $k$ is a positive integer not divided by $m$, and

$$
s \in\left\{\frac{1}{m}, \frac{2}{m}, \ldots, \frac{\operatorname{deg} P+1}{m}\right\} .
$$

4. For any positive integer $n$ and a polynomial $P$ the function $L_{P}(1-n, Q)$ is a polynomial of degree $\operatorname{deg} P+m(n-1)+1$. Its coefficients are rational linear combinations of the coefficients of $P$.

5. For any positive integer $n$

$$
L_{P}^{\prime}(1-n, Q)=L_{P(t) Q(-t)^{n-1}}^{\prime}(0, Q),
$$

and (compare with [Va], Prop. 3.1 )

$$
\begin{aligned}
& L_{P}^{\prime}(0, Q)-\sum_{i=1}^{m} \log \Gamma_{P}\left(u_{i}\right)=\sum_{n=0}^{\infty} \frac{(-1)^{n}}{n !} \zeta^{\prime}(-n) \sum_{i=1}^{m} P^{(n)}\left(u_{i}\right)+\sum_{i=1}^{m} \star P\left(u_{i}\right) \\
& (20) \quad+\left.\frac{1}{2 m} \sum_{n=1}^{\infty} \frac{P^{(n)}(1)}{n !} \frac{(-1)^{n}}{(n+1) !} \frac{d^{n+1}}{d t^{n+1}} \log ^{2}\left(t^{m} Q(1 / t-1)\right)\right|_{t=0},
\end{aligned}
$$

where $Q\left(-u_{i}\right)=0$ and $\star P(u)=\sum_{n=1}^{\infty} \frac{(u-1)^{n+1}}{(n+1) !}\left(1+\frac{1}{2}+\cdots+\frac{1}{n}\right) P^{(n)}(1)$. 
6. If $\operatorname{deg} P<m-1$ then the series (19) is absolutely convergent at $s=1$ and

$$
\begin{aligned}
& L_{P}(1, Q)= \\
& \quad-\sum_{i=1}^{m}\left[\frac{P\left(u_{i}\right)}{Q^{\prime}\left(-u_{i}\right)} \frac{\Gamma^{\prime}\left(u_{i}\right)}{\Gamma\left(u_{i}\right)}+\sum_{n=1}^{\infty} \frac{(-1)^{n}}{n \cdot n !}\left(B_{n}\left(u_{i}\right)-\left(u_{i}-1\right)^{n}\right) \frac{P^{(n)}\left(u_{i}\right)}{Q^{\prime}\left(-u_{i}\right)}\right],
\end{aligned}
$$

where $Q\left(-u_{i}\right)=0$, and all roots of $Q$ are simple.

Remark. It was shown by M. Wodzicki, that the quadratic term in (20), the 'determinant anomaly', can be interpreted as a residue, see [Ka] and $[\mathrm{KV}]$.

Proof.

1. Straightforward.

2. Straightforward.

3. Consider the Taylor series of a function $L_{l}(s, Q)=\sum_{n=1}^{\infty} n^{l} Q(n)^{-s}$ at $Q(t)=t^{m}$. More explicitly, let $Q(n)=\left(n+u_{1}\right) \cdots\left(n+u_{m}\right)$ then

$$
Q(n)^{-s}=n^{-m s} \sum_{k_{1}, \ldots, k_{m}=0}^{\infty}\left(\begin{array}{c}
-s \\
k_{1}
\end{array}\right) \cdots\left(\begin{array}{c}
-s \\
k_{m}
\end{array}\right)\left(u_{1} / n\right)^{k_{1}} \cdots\left(u_{m} / n\right)^{k_{m}} .
$$

Thus we get an analytic continuation for $\Re s<(l+1) / m$ as a series

$$
L_{l}(s, Q)=\sum_{k_{1}, \ldots, k_{m}=0}^{\infty}\left(\begin{array}{c}
-s \\
k_{1}
\end{array}\right) \cdots\left(\begin{array}{c}
-s \\
k_{m}
\end{array}\right) u_{1}^{k_{1}} \cdots u_{m}^{k_{m}} \zeta\left(m s+k_{1}+\cdots+k_{m}-l\right)
$$

for any non-negative integer $l$. Linearity implies the rest.

4. Straightforward from the above expansion for $L_{l}(s, Q)$. (Note that values of the Riemann $\zeta$-function are rational at non-positive integers.)

5 . The first identity comes from the second property.

For the second one use the Taylor series of $L_{l}(s, Q)$ at $u_{1}=\cdots=u_{m}=0$ above and a well-known identity

$$
\left.\frac{d}{d s}(s \zeta(s+1))\right|_{s=0}=-\Gamma^{\prime}(1)=\gamma
$$

(coming from the functional equation of the Riemann $\zeta$-function). 
Then we get

$$
\begin{aligned}
& L_{l}^{\prime}(0, Q)=m \zeta^{\prime}(-l)+\sum_{\substack{k=1 \\
k \neq l+1}}^{\infty} \frac{(-1)^{k}}{k} \zeta(k-l)\left(u_{1}^{k}+\cdots+u_{m}^{k}\right)+ \\
& (-1)^{l+1} \gamma \frac{u_{1}^{l+1}+\cdots+u_{m}^{l+1}}{l+1}+\frac{(-1)^{l+1}}{m}(1+\cdots+1 / l) \frac{u_{1}^{l+1}+\cdots+u_{m}^{l+1}}{l+1}+ \\
& \frac{(-1)^{l+1}}{m} \sum_{1 \leq i<j \leq m} \sum_{k=1}^{l} \frac{u_{i}^{k}}{k} \cdot \frac{u_{j}^{l+1-k}}{l+1-k} .
\end{aligned}
$$

Now compare $L_{l}^{\prime}(0, Q)$ with $\sum_{i=1}^{m} \log \Gamma_{P}\left(u_{i}+1\right)$ for $P(t)=(1-t)^{l}$.

The definition of $\Gamma_{P}(u)$ once combined with a well-known Taylor expansion

$$
\log \Gamma(u+1)=-\gamma u+\sum_{m=2}^{\infty}(-1)^{m} \zeta(m) \frac{u^{m}}{m}
$$

gives

$$
\frac{d}{d u} \log \Gamma_{P}(u)=\frac{d}{d u} \mathfrak{Q} P(u)-\gamma P(u)+\sum_{m=1}^{\infty}(-1)^{m+1} \zeta(m+1)(u-1)^{m} P(u) .
$$

Finally, we identify $L_{(1-t)^{l}}^{\prime}(0, Q(t+1))$ with

$$
\begin{aligned}
& L_{l}^{\prime}(0, Q)= \\
& \sum_{i=1}^{m} \log \Gamma_{(1-t)^{l}}\left(u_{i}+1\right)+(-1)^{l}\left(1+\frac{1}{2}+\cdots+\frac{1}{l}\right) \frac{u_{1}^{l+1}+\cdots+u_{m}^{l+1}}{l+1}+ \\
& \sum_{k=0}^{l}(-1)^{k}\left(\begin{array}{l}
l \\
k
\end{array}\right) \zeta^{\prime}(k-l)\left(u_{1}^{k}+\cdots+u_{m}^{k}\right)+\frac{(-1)^{l+1}}{2 m} \sum_{1 \leq i, j \leq m} \sum_{k=1}^{l} \frac{u_{i}^{k}}{k} \cdot \frac{u_{j}^{l+1-k}}{l+1-k}, \\
& \text { and } L_{P}^{\prime}(0, Q)=\sum_{l=0}^{\infty} \frac{(-1)^{l}}{l !} P^{(l)}(1) L_{l}^{\prime}(0, Q(t-1)) .
\end{aligned}
$$

6. The convergence is straightforward.

To compute the value of $L_{P}(1, Q)$, consider a one-parameter variation of the series (19), namely, $L_{P}(s, Q+\tau)$. Clearly,

$$
\frac{\partial}{\partial \tau} L_{P}^{\prime}(s, Q+\tau)=-s L_{P}^{\prime}(s+1, Q+\tau)-L_{P}(s+1, Q+\tau) .
$$

Now specialize $s$ and $\tau$ to 0 . We get

$$
\left.\frac{\partial}{\partial \tau} L_{P}^{\prime}(0, Q+\tau)\right|_{\tau=0}=-L_{P}(1, Q)
$$


Then use the formula (20):

$$
\begin{aligned}
& \frac{\partial}{\partial \tau} L_{P}^{\prime}(0, Q+\tau)=\sum_{i=1}^{m} \frac{\Gamma_{P}^{\prime}\left(u_{i}(\tau)\right)}{\Gamma_{P}\left(u_{i}(\tau)\right)} u_{i}^{\prime}(\tau)+ \\
& \sum_{n=0}^{\infty} \frac{(-1)^{n}}{n !} \zeta^{\prime}(-n) \sum_{i=1}^{m} P^{(n+1)}\left(u_{i}(\tau)\right) \cdot u_{i}^{\prime}(\tau)+\sum_{i=1}^{m} u_{i}^{\prime}(\tau) \cdot \frac{\partial}{\partial \tau} \star P\left(u_{i}(\tau)\right) \\
& +\left.\frac{1}{2 m} \sum_{n=1}^{\infty} \frac{P^{(n)}(1)}{n !} \frac{(-1)^{n}}{(n+1) !} \frac{d^{n+1}}{d t^{n+1}} \frac{\partial}{\partial \tau} \log ^{2}\left(t^{m}(Q(1 / t-1)+\tau)\right)\right|_{t=0} .
\end{aligned}
$$

Under the assumption on degree of the polynomial $P$, the latter sum is trivial, since

$$
\frac{\partial}{\partial \tau} \log ^{2}\left(t^{m}(Q(1 / t-1)+\tau)\right)=\left(\frac{2 \log \left(t^{m}(Q(1 / t-1)+\tau)\right)}{t^{m}(Q(1 / t-1)+\tau)}\right) t^{m} .
$$

Clearly, $u_{i}^{\prime}(\tau)=1 / Q^{\prime}\left(-u_{i}(\tau)\right)$, in particular, $u_{i}^{\prime}(0)=1 / Q^{\prime}\left(-u_{i}\right)$, when $-u_{i}$ is a simple root of $Q$. We use the formula (22) below to find

$$
\frac{d}{d u} \star=\sum_{m=1}^{\infty}(-1)^{m+1} \frac{(u-1)^{m}}{m \cdot m !} \frac{d^{m}}{d u^{m}} .
$$

Finally we get the desired identity.

The operator $\star$ admits more descriptions:

\section{Lemma 4.3.}

1. Integral formula:

$$
\star \varphi(u)=\frac{1}{2} \int_{1}^{u} \int_{1}^{u} \frac{\varphi(v)-\varphi(t)}{v-t} d t d v=\int_{1}^{u} \int_{1}^{v} \frac{\varphi(v)-\varphi(t)}{v-t} d t d v .
$$

2.

$$
\star=\sum_{m=1}^{\infty}(-1)^{m+1}\left(1+\frac{1}{2}+\cdots+\frac{1}{m}\right) \frac{(u-1)^{m+1}}{(m+1) !} \frac{d^{m}}{d u^{m}} .
$$

3.

$$
\star=\sum_{m=1}^{\infty}(-1)^{m+1} \star\left(\begin{array}{c}
u-1 \\
m
\end{array}\right)\left(1-\mathfrak{T}^{-1}\right)^{m},
$$

where $\star$ can actually be replaced by any operator $\star=\sum_{n=1}^{\infty} A_{n} \frac{(u-1)^{n+1}}{(n+1) !} \frac{d^{n}}{d u^{n}}$ such that $\star=\left.\sum_{n=1}^{\infty}(-1)^{n+1} A_{n} \frac{(u-1)^{n+1}}{(n+1) !} \frac{d^{n}}{d t^{n}}\right|_{t=1}$. 
Proof.

1. Replace $\varphi(v)$ and $\varphi(t)$ by their Taylor series at $v=1$ and $t=1$ respectively. This proves the first identity.

2. Replace $\varphi(v)$ and $\varphi(t)$ in the integral by their Taylor series at $v=u$ and $t=u$ respectively. This proves the second identity.

3. To prove the third one, notice that $\mathfrak{T}=\exp d / d u$, so $d^{m} / d u^{m}$ and $\left(1-\mathfrak{T}^{-1}\right)^{m}$ form two bases for the same space of "pro-differential" operators with constant coefficients. Then $\star=\sum_{m=1}^{\infty} P_{m}(u)\left(1-\mathfrak{T}^{-1}\right)^{m}$ for some polynomials $P_{m}(u)$.

Denote $d / d u$ by $x$ and $1-\mathfrak{T}^{-1}$ by $N$. Then

$$
P_{m}(u)=\sum_{n=1}^{\infty} A_{n} \frac{(u-1)^{n+1}}{(n+1) !} \operatorname{res}_{N=0} \frac{x^{n} d N}{N^{m+1}} .
$$

Notice, that $r e s_{N=0} \frac{x^{n} d N}{N^{m+1}}=(-1)^{n} \frac{d^{n}}{d t^{n}} \operatorname{res}_{N=0} \frac{e^{-x(t-1)} d N}{N^{m+1}}$. By the assumption on $\star$ above

$$
P_{m}(u)=-\star \operatorname{res}_{N=0} \frac{\left(e^{-x}\right)^{u-1} d N}{N^{m+1}} .
$$

Since $N=1-e^{-x}$, the residue is $(-1)^{m}\left(\begin{array}{c}u-1 \\ m\end{array}\right)$ and $P_{m}(u)=(-1)^{m+1} \star$ $\left(\begin{array}{c}u-1 \\ m\end{array}\right)$.

\section{Acknowledgements}

This paper would never have been written without generous help of and encouragement from Alexander Goncharov. Discussions with, remarks, interest and/or criticism of Alexander Beilinson, B. H. Gross and Don Zagier gave me energy for some further work. I am also indebted to Andrey Levin, who had initiated my study of the subject, and Yu. I. Manin for his interest and emotional support during preparation of $[R]$.

\section{References}

[An] G. Anderson, The hyperadelic gamma function, Invent. Math., 95 (1989), 63131.

[Ar] E. Artin, The gamma function. Athena Series: Selected Topics in Mathematics, Holt, Rinehart and Winston, New York-Toronto-London 1964.

[Ba] E. W. Barnes, On the theory of multiple gamma functions, Trans. Cambridge Philos. Soc., 19 (1904), 374-425.

[BG] I. N. Bernstein, S. I. Gel'fand, Meromorphic properties of the functions $P^{\lambda}$, Funkt. Analiz i ego Prilozh., 3 (1969), 84-85.

[C-N1] P. Cassou-Noguès, Analogues p-adiques des fonctions $\Gamma$-multiples, Astérisque, 61 (1979), 43-55.

[C-N2] _ _ Valeurs aux entiers négatifs des séries de Dirichlet associées à un polynôme. I, J. Numb. Theory, 14 (1982), 32-64. 
[C-N3] , Valeurs aux entiers négatifs des séries de Dirichlet associées à un polynôme. II, Amer. J. Math. 106 (1984), 255-299.

[Den] Ch. Deninger, On the $\Gamma$-factors attached to motives, Invent. Math., 104 (1991), 245-261.

[DP] J. Dufresnoy and Ch. Pisot, Sur la relation fonctionelle $f(x+1)-f(x)=\varphi(x)$, Bull. Soc. Math. Belg., 15 (1963), 259-270.

[E] Erdélyi et al, Higher transcendental functions, vol. I. Bateman Manuscript Project, New York, McGraw-Hill, 1953.

[Ka] C. Kassel, Le r'esidu non commutatif d'apr'es M. Wodzicki, Séminair Bourbaki 708 (1989), 199-229.

[KV] M. Kontsevich, S. Vishik, Geometry of determinants of elliptic operators, a short version: Preprint hep-th/9406140.

[K1] N. Kurokawa, Gamma factors and Plancherel measures, Proc. Japan Acad. Sci., 68 (1992), 256-260.

[K2] _ Multiple zeta functions: an example, Adv. Stud. in Pure Math., 21 (1992), 219-226.

[M] Yu. I. Manin, Lectures on zeta functions and motives (according to Deninger and Kurokawa), S. M. F., Astérisque, 228 (1995).

[Me] H. J. Mellin, Eine Formel für den Logarithmus transcendenter Functionen von endlichten Gischlecht, Acta Soc. Scient. fennicae, 29 (1900).

[Mo] C. J. Moreno, The Chowla-Selberg formula, J. Numb. Theory, 228 (1983), 226-245.

[R] M. Rovinskiǔ, Meromorphic functions connected to the polylogarithms, Funkt. Anal. Pril. (in Russian), 25 (1991), 88-91.

[Va] I. Vardi, Determinants of laplacians and multiple gamma functions, S.I.A.M. J. Math. Anal., 19 (1988), 493-507.

[Vi] M.-F. Vignéras, L'equation fonctionelle de la fonction zêta de Selberg du group modulaire PSL $(2, \mathbb{Z})$, Astérisque, 61 (1979), 235-249.

[Vo] A. Voros, Spectral functions and Selberg zeta functions, Comm. Math. Phys., 111 (1987), 439-465.

[W] A. Weil, Elliptic functions according to Eisenstein and Kronecker. Ergebnisse der Mathematik und ihrer Grenzgebiete, Band 88. Springer-Verlag, Berlin-New York, 1976.

[WW] E. T. Whittaker and G. N. Watson. A course of modern analysis, Cambridge, 1962.

Independent University of Moscow, Moscow, RUSSIA, and MaX-PlanckInstitut FÜr MATHEMATIK, BonN, GERMANY

E-mail address: marat@mail.dnttm.ras.ru 